TRANSACTIONS OF THE

AMERICAN MATHEMATICAL SOCIETY

Volume 351, Number 3, March 1999, Pages 985-995

S 0002-9947(99)02053-X

\title{
DUAL KINEMATIC FORMULAS
}

\author{
GAOYONG ZHANG
}

Dedicated to Professor De-lin Ren on the occasion of his 65th birthday

\begin{abstract}
We establish kinematic formulas for dual quermassintegrals of star bodies and for chord power integrals of convex bodies by using dual mixed volumes. These formulas are extensions of the fundamental kinematic formula involving quermassintegrals to the cases of dual quermassintegrals and chord power integrals. Applications to geometric probability are considered.
\end{abstract}

\section{INTRODUCTION}

Let $K$ be a convex body in $\mathbb{R}^{n}$. Denote by $\operatorname{vol}_{i}(K \mid \xi)$ the $i$-dimensional volume of the projection of $K$ onto an $i$-dimensional subspace $\xi \subset \mathbb{R}^{n}$. The important geometric invariants related to the projection of convex bodies are the quermassintegrals defined by

$$
W_{n-i}(K)=\frac{\omega_{n}}{\omega_{i}} \int_{\xi \in G_{i, n}} \operatorname{vol}_{i}(K \mid \xi) d \xi, \quad 0 \leq i \leq n,
$$

where the Grassmann manifold $G_{i, n}$ is endowed with the normalized Haar measure, and $\omega_{n}$ is the volume of the unit ball $B_{n}$ in $\mathbb{R}^{n}$. The quermassintegrals are generalizations of the surface area and the volume. Indeed, $n W_{1}(K)$ is the surface area of $K$, and $W_{0}(K)$ is the volume of $K$.

The quermassintegrals arise in many areas of mathematics and have different definitions. If $K$ has a $C^{2}$ boundary, they are the integrals of elementary symmetric functions of the principal curvatures over the boundary. In the theory of mixed volumes, the quermassintegrals are called simple mixed volumes. They are also called projection measures, intrinsic volumes, etc. The reader should consult [San] and $[\mathrm{Sch}]$ for the details.

Let $G(n)$ be the group of rigid motions in $\mathbb{R}^{n}$. The beautiful integral geometric formula of convex bodies $K$ and $L$ states that

$$
\int_{g \in G(n)} \chi(K \cap g L) d \mu(g)=\frac{1}{\omega_{n}} \sum_{i=0}^{n}\left(\begin{array}{l}
n \\
i
\end{array}\right) W_{i}(K) W_{n-i}(L),
$$

where $\chi$ is the Euler characteristic and $\mu$ is the invariant measure of $G(n)$ whose restriction to the rotation group is the invariant probability measure and the restriction to the translation group is the Lebesgue measure. The formula (1.1) is

Received by the editors September 13, 1996 and, in revised form, December 2, 1996.

1991 Mathematics Subject Classification. Primary 52A22; Secondary 53C65, 60D05.

Key words and phrases. Kinematic formula, dual quermassintegral, chord power integral, dual mixed volume, star body, convex body, geometric probability.

Research supported partially by NSF Grant DMS-9504913.

(C)1999 American Mathematical Society 
called the fundamental kinematic formula (see [San], p. 267). For the history and various generalizations of the fundamental kinematic formula to submanifolds in homogeneous spaces, one should consult $[\mathrm{H}]$. See $[\mathrm{Sch}]$ for generalizations to nonsmooth objects and more references. In this paper, we establish kinematic formulas for other important geometric invariants instead of quermassintegrals. It is shown that the dual quermassintegrals and the chord power integrals share the same kind of formulas as the quermassintegrals.

The dual quermassintegrals of a star body $K$ are defined by

$$
\tilde{W}_{n-i}(K)=\frac{\omega_{n}}{\omega_{i}} \int_{\xi \in G_{i, n}} \operatorname{vol}_{i}(K \cap \xi) d \xi, \quad 0 \leq i \leq n .
$$

While the quermassintegrals are connected with the projections of convex bodies, the dual quermassintegrals are closely related to the cross sections of star bodies. It is shown in $[\mathrm{K}]$ that they are the only rotation invariant continuous star valuations with the corresponding homogeneity.

The dual quermassintegrals coincide with the dual simple mixed volumes. Dual mixed volumes are the counterparts of mixed volumes. While the mixed volumes date back to Minkowski in the last century, the dual mixed volumes were only recently discovered. They play the roles in the study of cross-sections of convex bodies as the mixed volumes do for the study of projections of convex bodies. However, this duality is not at all trivial. One should read the book [G4] for an excellent illustration. Dual mixed volumes are far from well understood. Their applications to the characterizations of intersection bodies and the solution of the Busemann-Petty problem are very recent developments. See [G1]-[G3], [L], and [Z1]. In this paper, we use dual mixed volumes to establish kinematic formulas for dual quermassintegrals. The following formula is the counterpart of the fundamental kinematic formula for dual quermassintegrals.

Let $K$ and $L$ be star bodies with respect to the origin $O$ in $\mathbb{R}^{n}$. If $N_{g}$ is the line segment joining $O$ and $g O$ for $g \in G(n)$, then there is the dual fundamental kinematic formula

$$
\int_{g \in G(n)} \chi\left(K \cap g L \cap N_{g}\right) d \mu(g)=\frac{1}{\omega_{n}} \sum_{i=0}^{n}\left(\begin{array}{c}
n \\
i
\end{array}\right) \tilde{W}_{i}(K) \tilde{W}_{n-i}(L) .
$$

Denote by $A G_{k, n}$ the affine Grassmann manifold of $k$-dimensional planes in $\mathbb{R}^{n}$. Let $d \xi_{k}$ be the normalized invariant measure of $A G_{k, n}$ whose restriction to the Grassmann manifold $G_{k, n}$ is the invariant probability measure. Let $K$ be a convex body in $\mathbb{R}^{n}$, and let $\xi_{1}$ be a random line intersecting $K$. Then $\operatorname{vol}_{1}\left(K \cap \xi_{1}\right)$ is the chord length of the intersection $K \cap \xi_{1}$. The chord power integrals of $K$ are defined by

$$
I_{m}(K)=\frac{2 \alpha_{n-1}}{n} \int_{\xi_{1} \in A G_{1, n}} \operatorname{vol}_{1}\left(K \cap \xi_{1}\right)^{m} d \xi_{1}, \quad 0 \leq m<\infty,
$$

where $\alpha_{n-1}$ is the surface area of the unit sphere $S^{n-1}$. The chord power integrals are also generalizations of the surface area and the volume. They have the following relation with the integrals of square of volumes of higher dimensional sections of convex bodies:

$$
I_{k+1}(K)=\frac{2(k+1) \omega_{n}}{\omega_{k}} \int_{\xi_{k} \in A G_{k, n}} \operatorname{vol}_{k}\left(K \cap \xi_{k}\right)^{2} d \xi_{k} .
$$


They are also closely related to the dual quermassintegrals and integrals of power of distance of points in convex bodies. We prove by (1.2) that the chord power integrals satisfy the following kinematic formula.

Let $x$ and $y$ be random points in convex bodies $K$ and $L$ in $\mathbb{R}^{n}$, respectively. If $N_{g}$ is the line segment joining $x$ and $g y$ for $g \in G(n)$, then

$$
\int_{\substack{x \in K, y \in L \\
g \in G(n)}} \chi\left(K \cap g L \cap N_{g}\right) d \mu(g) d x d y=\frac{1}{\omega_{n}} \sum_{i=0}^{n}\left(\begin{array}{c}
n \\
i
\end{array}\right) \frac{I_{n-i+1}(K) I_{i+1}(L)}{(n-i+1)(i+1)} .
$$

We apply the formulas (1.1) and (1.3) to study a problem in geometric probability. Given two convex bodies in $\mathbb{R}^{n}$ which have nonempty intersection. One is random, another is fixed. In general, the union of the two convex bodies is not convex. How close is it to be convex? In precise terms, let $K$ be a fixed convex body and $L$ be a random convex body in $\mathbb{R}^{n}$ satisfying $K \cap L \neq \emptyset$. If $x \in K$ and $y \in L$ are randomly chosen, what is the probability that the line segment joining $x$ and $y$ is contained in the union $K \cup L$ ? We show that this conditional probability attains its maximum at balls when the volumes of the convex bodies are given. Computation also shows that this probability approaches zero as the dimension becomes large.

\section{Kinematic Formulas of DUAL QUERMASSINTEGRALS}

Let $K$ be a star-shaped compact set with respect to the point $z$ in $\mathbb{R}^{n}$. The radial function $\rho_{K}$ is defined, for all $u \in S^{n-1}$ such that the line through $z$ parallel to $u$ intersects $K$, by

$$
\rho_{K}(z, u)=\max \{c: z+c u \in K\} .
$$

When $z$ is the origin, we usually denote $\rho_{K}(o, u)$ by $\rho_{K}(u)$. By a star body we mean a star-shaped compact set $K$ whose radial function $\rho_{K}(z, u)$ is continuous with respect to $u$.

There is the formula for the dual quermassintegrals,

$$
\tilde{W}_{n-i}(K)=\frac{1}{n} \int_{S^{n-1}} \rho_{K}^{i}(u) d u, \quad 0 \leq i \leq n .
$$

If $K$ is a star body with respect to $z$, the following is a slight extension of the quermassintegrals:

$$
\tilde{W}_{n-r}(K, z)=\frac{1}{n} \int_{S^{n-1}} \rho_{K}^{r}(z, u) d u, \quad r \in \mathbb{R} .
$$

For star bodies $K, L$ with respect to $z \in \mathbb{R}^{n}$ and positive numbers $a, b$, the radial linear combination $a K \tilde{+} b L$ is a star body defined by

$$
\rho_{a K \tilde{+} b L}(z, u)=a \rho_{K}(z, u)+b \rho_{L}(z, u) .
$$

The volume of the radial sum $a K \tilde{+} b L$ has the expansion

$$
V(a K \tilde{+} b L)=\sum_{i=0}^{n}\left(\begin{array}{c}
n \\
i
\end{array}\right) \tilde{V}_{i}(K, L) a^{n-i} b^{i} .
$$

The coefficients $\tilde{V}_{i}(K, L)$ are called the dual mixed volumes of $K$ and $L$. They satisfy the formula

$$
\tilde{V}_{i}(K, L)=\frac{1}{n} \int_{S^{n-1}} \rho_{K}^{n-i}(z, u) \rho_{L}^{i}(z, u) d u .
$$

For the details of the theory of dual mixed volumes, see [L], [G4], [K], and [Z1]. 
Theorem 2.1. Let $K$ and $L$ be star bodies with respect to the origin $O$ in $\mathbb{R}^{n}$. If $N_{g}$ is the line segment joining $O$ and $g O$ for $g \in G(n)$, then there is the following dual fundamental kinematic formula:

$$
\int_{g \in G(n)} \chi\left(K \cap g L \cap N_{g}\right) d \mu(g)=\frac{1}{\omega_{n}} \sum_{i=0}^{n}\left(\begin{array}{c}
n \\
i
\end{array}\right) \tilde{W}_{i}(K) \tilde{W}_{n-i}(L) .
$$

Proof. Let $\nu$ be the invariant probability measure on $S O(n)$. First, we show the formula

$$
\int_{e \in S O(n)} \tilde{V}_{i}(K, e L) d \nu(e)=\frac{1}{\omega_{n}} \tilde{W}_{i}(K) \tilde{W}_{n-i}(L) .
$$

Since $S O(n)$ acts on $S^{n-1}$ transitively, (2.1) gives

$$
\begin{aligned}
\int_{e \in S O(n)} \rho_{e L}^{i}(u) d \nu(e) & =\int_{e \in S O(n)} \rho_{L}^{i}\left(e^{-1} u\right) d \nu(e)=\frac{1}{\alpha_{n-1}} \int_{u \in S^{n-1}} \rho_{L}^{i}(u) d u \\
& =\frac{n}{\alpha_{n-1}} \tilde{W}_{n-i}(L)=\frac{1}{\omega_{n}} \tilde{W}_{n-i}(L) .
\end{aligned}
$$

By (2.3), it follows that

$$
\begin{aligned}
\int_{e \in S O(n)} \tilde{V}_{i}(K, e L) d \nu(e) & =\int_{e \in S O(n)} d \nu(e) \frac{1}{n} \int_{S^{n-1}} \rho_{K}^{n-i}(u) \rho_{e L}^{i}(u) d u \\
& =\frac{1}{n} \int_{S^{n-1}} \frac{1}{\omega_{n}} \rho_{K}^{n-i}(u) \tilde{W}_{i}(L) d u=\frac{1}{\omega_{n}} \tilde{W}_{i}(K) \tilde{W}_{n-i}(L) .
\end{aligned}
$$

This shows (2.5).

For a motion $g \in G(n)$, let $Q=g O$. Then $Q$ is a fixed point of $g L$ when $L$ is moved by $g$, and $N_{g}$ is the line segment $\overline{O Q}$ joining $O$ and $Q$. Let $d Q$ be the volume element of $\mathbb{R}^{n}$ at $Q$. If the motion $g$ is given by the rotation $e$ and the translation $O \rightarrow Q$, then the invariant measure $\mu$ on $G(n)$ is given by

$$
d \mu(g)=d Q d \nu(e)
$$

It is easy to see the equivalence

$$
\chi\left(K \cap g L \cap N_{g}\right)=1 \Longleftrightarrow Q \in K \tilde{+}(-e L) .
$$

From (2.2) and (2.5)-(2.7), we have

$$
\begin{aligned}
\int_{g \in G(n)} \chi & \left(K \cap g L \cap N_{g}\right) d \mu(g)=\int_{g \in G(n)} \chi\left(K \cap g L \cap N_{g}\right) d Q d \nu(e) \\
& =\int_{e \in S^{n-1}} V(K \tilde{+}(-e L)) d \nu(e)=\int_{e \in S^{n-1}} \sum_{i=0}^{n}\left(\begin{array}{c}
n \\
i
\end{array}\right) \tilde{V}_{i}(K,-e L) d \nu(e) \\
& =\frac{1}{\omega_{n}} \sum_{i=0}^{n}\left(\begin{array}{c}
n \\
i
\end{array}\right) \tilde{W}_{i}(K) \tilde{W}_{n-i}(L) . \quad
\end{aligned}
$$

Let $K$ and $L$ be star bodies with respect to the origin. There is the formula (see [San], p. 259)

$$
\int_{g \in G(n)} V(K \cap g L) d \mu(g)=V(K) V(L) .
$$

This formula is even true for compact sets. It is the mean value formula of the intersection volume $V(K \cap g L)$. What is the mean value formula for the intersection 
length $\operatorname{vol}_{1}\left(K \cap g L \cap N_{g}\right)$ ? The following theorem says that it can be expressed by the dual quermassintegrals.

Theorem 2.2. Let $K$ and $L$ be star bodies with respect to the origin $O$ in $\mathbb{R}^{n}$. If $N_{g}$ is the line segment joining $O$ and $g O$ for $g \in G(n)$, then

$$
\int_{g \in G(n)} \operatorname{vol}_{1}\left(K \cap g L \cap N_{g}\right) d \mu(g)=\frac{1}{(n+1) \omega_{n}} \sum_{i=1}^{n}\left(\begin{array}{c}
n+1 \\
i
\end{array}\right) \tilde{W}_{n-i}(K) \tilde{W}_{i-1}(L) .
$$

Proof. For a motion $g \in G(n)$, let $Q=g O$ and $e$ be the rotation induced by $g$. Then we have (2.6). We first show the formula

$$
\int \operatorname{vol}_{1}\left(K \cap g L \cap N_{g}\right) d Q=\frac{1}{n(n+1)} \sum_{i=1}^{n}\left(\begin{array}{c}
n+1 \\
i
\end{array}\right) \int_{S^{n-1}} \rho_{K}^{i}(u) \rho_{e L}^{n-i+1}(-u) d u .
$$

Note that the integration on the left hand side of (2.9) can be restricted to the star body $K \tilde{+}(-e L)$. Let

$$
\begin{array}{cl}
\rho=|\overrightarrow{O Q}|, & u=\frac{1}{\rho} \overrightarrow{O Q} \\
\rho_{M}=\max \left\{\rho_{K}(u), \rho_{e L}(-u)\right\}, & \rho_{m}=\min \left\{\rho_{K}(u), \rho_{e L}(-u)\right\} .
\end{array}
$$

Then, we have

$$
\operatorname{vol}_{1}\left(K \cap g L \cap N_{g}\right)= \begin{cases}\rho, & \rho \leq \rho_{m} \\ \rho_{m}, & \rho_{m}<\rho \leq \rho_{M} \\ \rho_{m}+\rho_{M}-\rho, & \rho_{M}<\rho \leq \rho_{m}+\rho_{M}\end{cases}
$$

This gives

$$
\begin{aligned}
& \int \operatorname{vol}_{1}\left(K \cap g L \cap N_{g}\right) \rho^{n-1} d \rho \\
& \quad=\int_{0}^{\rho_{m}} \rho \rho^{n-1} d \rho+\int_{\rho_{m}}^{\rho_{M}} \rho_{m} \rho^{n-1} d \rho+\int_{\rho_{M}}^{\rho_{m}+\rho_{M}}\left(\rho_{m}+\rho_{M}-\rho\right) \rho^{n-1} d \rho \\
& =\frac{1}{n(n+1)}\left(\left(\rho_{m}+\rho_{M}\right)^{n+1}-\rho_{m}^{n+1}-\rho_{M}^{n+1}\right) \\
& =\frac{1}{n(n+1)} \sum_{i=1}^{n}\left(\begin{array}{c}
n+1 \\
i
\end{array}\right) \rho_{m}^{n-i+1} \rho_{M}^{i} \\
& =\frac{1}{n(n+1)} \sum_{i=1}^{n}\left(\begin{array}{c}
n+1 \\
i
\end{array}\right) \rho_{K}^{i}(u) \rho_{e L}^{n-i+1}(-u) .
\end{aligned}
$$

Thus, we obtain (2.9) by the spherical coordinates. 
From (2.6) and (2.9), we have

$$
\begin{aligned}
\int_{g \in G(n)} \operatorname{vol}_{1}\left(K \cap g L \cap N_{g}\right) d \mu(g) \\
=\int_{e \in S O(n)}\left(\int \operatorname{vol}_{1}\left(K \cap g L \cap N_{g}\right) d Q\right) d \nu(e) \\
=\int_{e \in S O(n)}\left(\frac{1}{n(n+1)} \sum_{i=1}^{n}\left(\begin{array}{c}
n+1 \\
i
\end{array}\right) \int_{S^{n-1}} \rho_{K}^{i}(u) \rho_{e L}^{n-i+1}(-u) d u\right) d \nu(e) \\
=\frac{1}{n(n+1)} \sum_{i=1}^{n}\left(\begin{array}{c}
n+1 \\
i
\end{array}\right) \int_{S^{n-1}} \rho_{K}^{i}(u) \int_{e \in S O(n)} \rho_{L}^{n-i+1}\left(-e^{-1} u\right) d \nu(e) d u \\
=\frac{1}{n+1} \sum_{i=1}^{n}\left(\begin{array}{c}
n+1 \\
i
\end{array}\right) \tilde{W}_{n-i}(K) \frac{n}{\alpha_{n-1}} \tilde{W}_{i-1}(L) \\
=\frac{1}{(n+1) \omega_{n}} \sum_{i=1}^{n}\left(\begin{array}{c}
n+1 \\
i
\end{array}\right) \tilde{W}_{n-i}(K) \tilde{W}_{i-1}(L) . \quad
\end{aligned}
$$

Before considering the chord power integrals, we give more integral formulas for the dual quermassintegrals. Let $A G_{n-i, n}$ be the set of $(n-i)$-dimensional affine subspaces in $\mathbb{R}^{n}$. It is a homogeneous space under the action of the motion group $G(n)$ (see [San], p.199). For $\zeta \in A G_{n-i, n}$, let $\xi=\zeta^{\perp}$, the $i$-dimensional subspace orthogonal to $\zeta$ in $\mathbb{R}^{n}$, and let $Q=\xi \cap \zeta$. Then $\xi \in G_{i, n}, Q \in \xi$. If $d \xi$ is the $S O(n)$-invariant probability measure on $G_{i, n}$ and $d Q$ is the volume element of $\xi$ at $Q$, then

$$
d \zeta=d Q d \xi
$$

is a $G(n)$-invariant measure of $A G_{n-i, n}$ (see [San], p. 204).

For a convex body $K$ in $\mathbb{R}^{n}$, the Crofton-Santaló formula states that (see [San], p. 245)

$$
\int_{A G_{n-i, n}} \operatorname{vol}_{n-i}(K \cap \zeta) d \zeta=V(K) .
$$

We give a similar formula involving dual quermassintegrals.

Proposition 2.3. Let $K$ be a star body with respect to the origin $O$ in $\mathbb{R}^{n}$. If $N_{\zeta}$ is the line segment joining $O$ and $Q=\zeta \cap \zeta^{\perp}$ for $F \in A G_{n-i, n}$, then

$$
\int_{\substack{Q \in K \\ \zeta \in A G_{n-i, n}}} \operatorname{vol}_{1}\left(K \cap N_{\zeta}\right)^{k} d \zeta=\frac{\alpha_{n-i}}{\omega_{n}} \tilde{W}_{n-i-k}(K), \quad i+k \geq 0 .
$$

Proof. From (2.10), we have

$$
\begin{aligned}
\int_{\substack{Q \in K \\
\zeta \in G_{n-i, n}}} \operatorname{vol}_{1}\left(K \cap N_{\zeta}\right)^{k} d \zeta=\int_{\substack{Q \in K \cap \xi \\
\xi \in G_{i, n}}} \operatorname{vol}_{1}\left(K \cap N_{\zeta}\right)^{k} d Q d \xi \\
=\int_{\substack{u \in S^{n-1} \cap \xi \\
\xi \in G_{i, n}}} \rho_{K \cap \xi}^{k+i}(u) d u d \xi=\frac{\alpha_{i-1}}{\alpha_{n-1}} \int_{S^{n-1}} \rho_{K}^{k+i}(u) d u \\
=\frac{n \alpha_{n-i}}{\alpha_{n-1}} \tilde{W}_{n-i-k}(K)=\frac{\alpha_{n-i}}{\omega_{n}} \tilde{W}_{n-i-k}(K) . \quad \square
\end{aligned}
$$


Let $S L(n)$ be the special linear group. It acts on $\mathbb{R}^{n}-\{O\}$ transitively. If $A(n, n-1)$ is the set of hyperplanes that do not go through the origin, then it is a homogeneous space under the action of $S L(n)$. For $\zeta \in A(n, n-1)$, let $Q=\zeta \cap \zeta^{\perp}$, $N_{\zeta}$ be the line segment joining $O$ and $Q, r$ be the length of $N_{\zeta}$, and $u=\frac{1}{r} \overrightarrow{O Q}$. The space $A(n, n-1)$ has an $S L(n)$-invariant measure which can be expressed as (see [San], p. 185)

$$
d \zeta=r^{-n-1} d r d u
$$

where $d r$ and $d u$ are the Lebesgue measures on $\mathbb{R}$ and $S^{n-1}$ respectively.

Proposition 2.4. Let $K$ be a star body with respect to the origin $O$ in $\mathbb{R}^{n}$. If $N_{\zeta}$ is the line segment joining $O$ and $Q=\zeta \cap \zeta^{\perp}$ for $\zeta \in A(n, n-1)$, then

$$
\int_{\substack{Q \in K \\ \zeta \in A(n, n-1)}} \operatorname{vol}_{1}\left(K \cap N_{\zeta}\right)^{k} d \zeta=\frac{n}{k-n} \tilde{W}_{2 n-k}(K), \quad k>n .
$$

Proof. From (2.11), we have

$$
\begin{array}{r}
\int_{\substack{Q \in K \\
\zeta \in A(n, n-1)}} \operatorname{vol}_{1}\left(K \cap N_{\zeta}\right)^{k} d \zeta=\int_{S^{n-1}}\left(\int_{0}^{\rho_{K}(u)} r^{k-n-1} d r\right) d u \\
=\frac{1}{k-n} \int_{S^{n-1}} \rho_{K}^{k-n}(u) d u=\frac{n}{k-n} \tilde{W}_{2 n-k}(K) . \quad \square
\end{array}
$$

Corollary 2.5. Let $K$ be a star body with respect to the origin $O$ in $\mathbb{R}^{n}$. If $N_{\zeta}$ is the line segment joining $O$ and $Q=\zeta \cap \zeta^{\perp}$ for $\zeta \in A(n, n-1)$, then

$$
\int_{\substack{Q \in K \\ \zeta \in A, n-1)}} \operatorname{vol}_{1}\left(K \cap N_{\zeta}\right)^{2 n} d \zeta=V(K) .
$$

\section{Kinematic FORMUlas OF CHORD POWER Integrals}

Let $K$ be a convex body in $\mathbb{R}^{n}$, and let $\xi_{1}$ be a random line intersecting $K$. Denote by $\sigma$ the chord length of the intersection $K \cap \xi_{1}, \sigma=\operatorname{vol}_{1}\left(K \cap \xi_{1}\right)$. The chord power integrals of $K$ are defined by

$$
I_{m}(K)=\frac{2 \alpha_{n-1}}{n} \int_{K \cap \xi_{1} \neq \emptyset} \sigma^{m} d \xi_{1}, \quad 0 \leq m<\infty,
$$

where $d \xi_{1}$ is the normalized invariant measure of the affine Grassmann manifold of lines, $A G_{1, n}$. Note that the normalization says that

$$
\int_{B_{n} \cap \xi_{1} \neq \emptyset} d \xi_{1}=\omega_{n-1}
$$

There are several interesting integral formulas for the chord power integrals (see [San]):

$$
\begin{aligned}
I_{0}(K) & =\frac{\omega_{n-1}}{n} S(K), \\
I_{1}(K) & =\frac{\alpha_{n-1}}{n} V(K), \\
I_{n+1}(K) & =(n+1) V(K)^{2} .
\end{aligned}
$$


The chord power integrals are connected with another set of integrals $J_{m}(K)$ given by

$$
J_{m}(K)=\int_{x, y \in K} r^{m} d x d y
$$

where $r$ is the distance between the points $x$ and $y$. There is the following formula between $I_{m}(K)$ and $J_{m}(K)$ :

$$
I_{m}(K)=\frac{m(m-1)}{n} J_{m-n-1}(K), \quad m>1 .
$$

When $m=n+1$, (3.4) yields (3.2). See [San], p. 238.

The chord power integrals are also connected with the dual quermassintegrals.

Lemma 3.1. Let $\tilde{W}_{n-k}(K, z)$ be the $(n-k)$ th dual quermassintegral of the convex body $K$ with respect to the point $z$ in $\mathbb{R}^{n}$. Then

$$
I_{k+1}(K)=(k+1) \int_{z \in K} \tilde{W}_{n-k}(K, z) d z, \quad-1<k<\infty .
$$

Proof. Let $\xi_{1}^{*}$ be an oriented line whose direction is $u \in S^{n-1}$. Let $z$ be a point in the convex body $K$. Denote by $t$ the distance of $z$ to the boundary of $K$ along the direction $u$. Suppose that $\xi_{1}^{*}$ passes through $z$. Then $d \xi_{1}^{*} d t=\alpha_{n-1}^{-1} d z d u$ (see [San], p. 207). Multiplying both sides by $t^{k}$ and integrating over $K$, we have

$$
\int_{K \cap \xi_{1}^{*} \neq \emptyset}\left(\int_{0}^{\sigma} t^{k} d t\right) d \xi_{1}^{*}=\alpha_{n-1}^{-1} \int_{z \in K}\left(\int_{S^{n-1}} t^{k} d u\right) d z
$$

This gives

$$
\frac{1}{k+1} \int_{K \cap \xi_{1}^{*} \neq \emptyset} \sigma^{k} d \xi_{1}^{*}=n \alpha_{n-1}^{-1} \int_{z \in K} \tilde{W}_{n-k}(K, z) d z, \quad-1<k<\infty .
$$

When $k=n$, formula (3.5) gives the Crofton-Hadwiger formula (3.2).

The chord power integrals are surprisingly related to the integrals of square of volumes of higher dimensional sections of convex bodies. Let $\xi_{k}$ be a $k$-dimensional plane in $\mathbb{R}^{n}$, and let $d \xi_{k}$ be the invariant measure of the affine Grassmann manifold $A G_{k, n}$. When restricted to $G_{k, n},\left.d \xi_{k}\right|_{G_{k, n}}=d \xi_{k}^{\circ}$ is the invariant probability measure on $G_{k, n}$.

Proposition 3.2. Let $K$ be a convex body in $\mathbb{R}^{n}$. Then

$$
I_{k+1}(K)=\frac{2(k+1) \omega_{n}}{\omega_{k}} \int_{K \cap \xi_{k} \neq \emptyset} \operatorname{vol}_{k}\left(K \cap \xi_{k}\right)^{2} d \xi_{k} .
$$

Proof. Let $z$ be a point in $K$. Suppose that $\xi_{k}$ passes through $z$. Denote by $d z$ the volume element of $\mathbb{R}^{n}$ at $z$, and denote by $d z^{(k)}$ the volume element of $\xi$ at $z$. Then

$$
d z^{(k)} d \xi_{k}^{*}=d \xi_{k}^{\circ} d z .
$$

See [San], p. 207. Multiplying both sides by $\operatorname{vol}_{k}\left(K \cap \xi_{k}\right)$ and integrating over $K$, we obtain

$$
\begin{aligned}
\int_{K \cap \xi_{k} \neq \emptyset} \int_{z \in K \cap \xi_{k}} \operatorname{vol}_{k}\left(K \cap \xi_{k}\right) d z^{(k)} d \xi_{k}^{*} & =\int_{z \in K} \int_{\xi_{k}^{\circ} \in G_{k, n}} \operatorname{vol}_{k}\left(K \cap \xi_{k}\right) d \xi_{k}^{\circ} d z, \\
2 \int_{K \cap \xi_{k} \neq \emptyset} \operatorname{vol}_{k}\left(K \cap \xi_{k}\right)^{2} d \xi_{k} & =\frac{\omega_{k}}{\omega_{n}} \int_{z \in K} \tilde{W}_{n-k}(K, z) d z .
\end{aligned}
$$

We complete the proof by Lemma 3.1 . 
The last proposition was proved first in [Z2] by a different method. See [R1], $\S 7.5 .4$, for more and related results.

Theorem 3.3. Let $K$ and $L$ be convex bodies in $\mathbb{R}^{n}$, and let $x$ and $y$ be random points in $K$ and $L$, respectively. If $N_{g}$ is the line segment joining $x$ and gy for $g \in G(n)$, then

$$
\int_{\substack{x \in K, y \in L \\
g \in G(n)}} \chi\left(K \cap g L \cap N_{g}\right) d \mu(g) d x d y=\frac{1}{\omega_{n}} \sum_{k=0}^{n}\left(\begin{array}{l}
n \\
k
\end{array}\right) \frac{I_{n-k+1}(K) I_{k+1}(L)}{(n-k+1)(k+1)} .
$$

Proof. Integrating the dual fundamental kinematic formula with respect to $x$ and $y$, we have

$$
\begin{aligned}
& \int_{\substack{x \in K, y \in L \\
g \in G(n)}} \chi\left(K \cap g L \cap N_{g}\right) d \mu(g) d x d y \\
& \quad=\frac{1}{\omega_{n}} \sum_{i=0}^{n}\left(\begin{array}{c}
n \\
i
\end{array}\right) \int_{K} \tilde{W}_{i}(K, x) d x \int_{L} \tilde{W}_{n-i}(L, y) d y .
\end{aligned}
$$

From this and Lemma 3.1, we obtain (3.6).

Similarly, from Theorem 2.2 and Lemma 3.1, we obtain

Theorem 3.4. Let $K$ and $L$ be convex bodies in $\mathbb{R}^{n}$, and let $x$ and $y$ be random points in $K$ and $L$, respectively. If $N_{g}$ is the line segment joining $x$ and gy for $g \in G(n)$, then

$$
\begin{aligned}
& \int_{\substack{x \in K, y \in L \\
g \in G(n)}} \operatorname{vol}_{1}\left(K \cap g L \cap N_{g}\right) d \mu(g) d x d y \\
& =\frac{1}{(n+1) \omega_{n}} \sum_{k=1}^{n}\left(\begin{array}{c}
n+1 \\
k
\end{array}\right) \frac{I_{n-k+2}(K) I_{k+1}(L)}{(n-k+2)(k+1)} .
\end{aligned}
$$

\section{Applichtions to geometric probability}

Consider two random convex bodies in $\mathbb{R}^{n}$ which have nonempty interestion. In general, the union of the two convex bodies is not convex. How close is the union to be convex? To be precise, one can let one convex body be fixed but another convex body intersect with the fixed body randomly. Let $K$ be a fixed convex body and $L$ be a random convex body in $\mathbb{R}^{n}$ satisfying $K \cap L \neq \emptyset$. Choose points $x \in K$ and $y \in L$ randomly. What is the probability that the line segment joining $x$ and $y$ is contained in the union $K \cup L$ ? Denote by $p_{n}(K, L)$ this conditional probability. From (1.1) and (1.3), we have

$$
\begin{aligned}
p_{n}(K, L) & =\frac{\int_{\substack{x \in K, y \in L \\
g \in G(n)}} \chi\left(K \cap g L \cap N_{g}\right) d \mu(g) d x d y}{\int_{\substack{x \in K, y \in L \\
g \in G(n)}} \chi(K \cap g L) d \mu(g) d x d y} \\
= & \frac{\sum_{k=0}^{n}\left(\begin{array}{l}
n \\
k
\end{array}\right) \frac{I_{n-k+1}(K) I_{k+1}(L)}{(n-k+1)(k+1)}}{\sum_{i=0}^{n}\left(\begin{array}{c}
n \\
i
\end{array}\right) W_{i}(K) W_{n-i}(L) V(K) V(L)} .
\end{aligned}
$$


Theorem 4.1. If $K$ and $L$ are convex bodies in $\mathbb{R}^{n}$ with the same volume, then

$$
p_{n}(K, L) \leq p_{n}\left(B_{n}, B_{n}\right)=\frac{4}{\omega_{n}^{2}} \sum_{k=0}^{n}\left(\begin{array}{l}
n \\
k
\end{array}\right) \frac{\omega_{2 n-k} \omega_{n+k}}{\alpha_{n-k} \alpha_{k}},
$$

with equality if and only if $K$ and $L$ are congruent balls.

proof. To show (4.2), we need two sets of inequalities. One is the generalizations of the isoperimetric inequality.

$$
W_{k}(K) \geq \omega_{n}\left(\frac{V(K)}{\omega_{n}}\right)^{\frac{n-k}{n}}, \quad 0 \leq k \leq n .
$$

See $[\mathrm{Sch}]$, p. 334. Another is the isoperimetric-type inequalities of chord power integrals.

$$
I_{k+1}(K) \leq I_{k+1}\left(B_{n}\right)\left(\frac{V(K)}{\omega_{n}}\right)^{\frac{n+k}{n}}, \quad 0 \leq k \leq n .
$$

The 2-dimensional case of the inequalities of chord power integrals is due to Blaschke (see [San], p. 238). The 3-dimensional case is due to $\mathrm{Wu}$ (see [R1], p. 215). The $n$-dimensional case is proved by Ren [R2] (see [R1], §7.5.3).

The chord power integrals of the unit ball $B_{n}$ are given by

$$
I_{k+1}\left(B_{n}\right)=\frac{2^{k+1} \omega_{n} \omega_{k+n}}{\omega_{k+1}} .
$$

See [R1], pp. 220 and 227, and [Z2]. Since the probability $p_{n}(K, L)$ is invariant under the dilations of convex bodies, that is, for any $\lambda>0$, one has

$$
p_{n}(\lambda K, \lambda L)=p_{n}(K, L),
$$

we can assume that $V(K)=V(L)=\omega_{n}$. Therefore, (4.1), (4.3) and (4.4) give that

$$
p_{n}(K, L) \leq \frac{1}{\omega_{n}^{4} 2^{n}} \sum_{k=0}^{n}\left(\begin{array}{l}
n \\
k
\end{array}\right) \frac{I_{n-k+1}\left(B_{n}\right) I_{k+1}\left(B_{n}\right)}{(n-k+1)(k+1)} .
$$

From the last inequality and (4.5), we obtain (4.2).

One can rewrite the maximum $p_{n}\left(B_{n}, B_{n}\right)$ in terms of Gamma functions.

$$
p_{n}\left(B_{n}, B_{n}\right)=\frac{1}{\pi} \Gamma\left(\frac{n}{2}+1\right)^{2} \sum_{k=0}^{n}\left(\begin{array}{l}
n \\
k
\end{array}\right) \frac{\Gamma\left(\frac{n-k+1}{2}\right) \Gamma\left(\frac{k+1}{2}\right)}{\Gamma\left(n-\frac{k}{2}+1\right) \Gamma\left(\frac{n+k}{2}+1\right)} .
$$

From this, it is very easy to compute special values of the maximum. The values approach to zero as the dimension becomes large.

\section{REFERENCES}

[G1] R.J. Gardner, Intersection bodies and the Busemann-Petty problem, Trans. Amer. Math. Soc. 342 (1994), 435-445. MR 94e:52008

[G2] - A positive answer to the Busemann-Petty problem in three dimensions, Annals of Math. 140 (1994), 435-447. MR 95i:52005

[G3] - On the Busemann-Petty problem concerning central sections of centrally symmetric convex bodies, Bull. Amer. Math. Soc. 30 (1994), 222-226. MR 94m:52007

[G4] - Geometric tomography, Cambridge University Press, Cambridge, 1995. MR 96j:52006

[H] R. Howard, The kinematic formula in Riemannian homogeneous spaces, Mem. Amer. Math. Soc. 106, 1993. MR 94d:53114 
[K] D. Klain, Star valuations and dual mixed volumes, Adv. Math. 121 (1996), 80-101. MR 97i:52009

[L] E. Lutwak, Intersection bodies and dual mixed volumes, Adv. Math. 71 (1988), 232-261. MR 90a:52023

[R1] De-lin Ren, Topics in Integral Geometry, World Scientific Publishing, River Edge, NJ, 1994. MR 96h:53087

[R2] - Two topics in integral geometry, Proceedings of the 1981 symposium on differential geometry and differential equations (Shanghai-Hefei), Science Press, Beijing, 1984. MR 87d:53124

[San] L.A. Santaló, Integral Geometry and Geometric Probability, Addison-Wesley, 1976. MR 55:6340

[Sch] R. Schneider, Convex Bodies: The Brunn-Minkowski Theory, Cambridge University Press, Cambridge, 1993. MR 94d:52007

[Z1] G. Zhang, Centered bodies and dual mixed volumes, Trans. Amer. Soc. 345 (1994), 777-801. MR 95d:52008

[Z2] Integral geometric inequalities, Acta Math. Sinica 34 (1991), 72-90. MR 92g:53066

School of Mathematics, Institute for Advanced Study and Department of Mathematics, University of Pennsylvania, Philadelphia, Pennsylvania 19104

Current address: Department of Mathematics, Polytechnic University, 6 Metrotech Center, Brooklyn, New York 11201

E-mail address: gzhang@math.poly.edu 